\title{
2D-ACAR SPECTRA OF INSULATING AND SUPERCONDUCTING Y-123*
}

\author{
L.C. Smedskjaer \\ Materials Science Division \\ Argonne National Laboratory, Argonne, IL 60439 \\ and \\ A. Bansil \\ Physics Department \\ Northeastern University, Boston, MA 02115

The submitted manuscript has been authored
by a contractor of the U.S. Government under
contract No. W-31-109-ENG-38. Accordingly,
the U.S. Government retains a nonexclusive,
royalty-free license to publish or reproduce the
published form of this contribution, or allow
others to do so, for U.S. Government purposes. \\ others to do so, for U.S. Government purposes.
}

J. Phys. Chem. Solids

jmc

\section{DISCLAIMER}

This report was prepared as an account of work sponsored by an agency of the United States Government. Neither the United States Government nor any agency thereof, nor any of their employees, makes any warranty, express or implied, or assumes any legal liability or responsibility for the accuracy, completeness, or usefulness of any information, apparatus, product, or process disclosed, or represents that its use would not infringe privately owned rights. Reference herein to any specific commercial product, process, or service by trade name, trademark, manufacturer, or otherwise does not necessarily constitute or imply its endorsement, recommendation, or favoring by the United States Government or any agency thereof. The views and opinions of authors expressed herein do not necessarily state or reflect those of the United States Government or any agency thereof.

\footnotetext{
${ }^{*}$ Work supported by the U.S. Department of Energy, BES-Materials Sciences under contract \#W-31-109-ENG-38 (LCS) including a subcontract to Northeastern University $(\mathrm{AB})$ and benefited from the allocation oif supercomputer time on th. NERSC and San Diego Supercompouter centers.
} 


\section{DISCLAIMER}

Portions of this document may be illegible in electronic image products. Images are produced from the best available original document. 


\title{
2D-ACAR SPECTRA OF INSULATING AND SUPERCONDUCTING Y-123
}

\author{
L.C. SMEDSKJAER ${ }^{1)}$ AND A. BANSI ${ }^{2)}$
}

1) Materials Science Division, Argonne National Laboratory, Argonne, IL 60439.

2) Physics Department, Northeastern University, Boston, Massachusetts 02115

\begin{abstract}
An overview of the two-dimensional angular correlation (2D-ACAR) positron annihilation results for the three fundamental phases of $\mathrm{YBa}_{2} \mathrm{Cu}_{3} \mathrm{O}_{x}$, namely, the normal metal, the superconductor, and the insulator, is presented. In addition to the c-axis projected momentum density, the recent results for the a-axis projection as well as the insulating Y123 are discussed. The experimental results are compared and contrasted with the corresponding band theory predictions as far as possible in order to gain insight into the electronic structure and Fermiology of this archetypal high- $T_{c}$ superconductor.
\end{abstract}

Keywords: positron annihilation spectroscopy, superconductivity, Fermi surface.

\section{INTRODUCTION}

Positron annihilation (2D-ACAR) studies of $\mathrm{YBa}_{2} \mathrm{Cu}_{3} \mathrm{O}_{7-\mathrm{x}}$ started immediately after the discovery of this archetypal high temperature superconductor [1-3]. The 2D-ACAR experiment is ideally suited to answer one of the fundamental questions that arose early, namely, whether the electron momentum density in the new materials possesses Fermi surface breaks like the conventional metals. Results in this regard would provide guidance in searching for a suitable theoretical description of the electronic structure, and thus help in developing an understanding of the mechanism responsible for the occurrence of high superconducting transition temperatures. More generally, the accuracy of any specific electronic structure model can in principle be assessed by comparing the predicted 2D-ACAR spectra with the corresponding observations, although such comparisons to date have been possible only for the local density (LDA) based band theory model, which is the only model where detailed theoretical predictions are currently available.

Despite some unresolved issues, the recent Argonne Fermiology workshop [4-6] marked a turning point in that, by combining the results from 2D-ACAR [7-11], ARPES [12-15], and dHvA [16,17] experiments, a general consensus was achieved regarding the existence of a Fermi surface in at least $\mathrm{YBa}_{2} \mathrm{Cu}_{3} \mathrm{O}_{7}$ in overall agreement with the band theory predictions. Concerning the 2D-ACAR spectroscopy in particular, extensive comparisons between the computed spectral intensities [18-22] and the measurements clearly establish the presence of the $\mathrm{Cu}-\mathrm{O}$ chain related ridge Fermi surface in $\mathrm{YBa}_{2} \mathrm{Cu}_{3} \mathrm{O}_{7}$; some evidence for other pieces of the Fermi surface is also indicated [11]. Further insight into the electronic structure of $\mathrm{Y}-123$ will obviously be obtained by augmenting the studies of the metallic phase with those of the insulating phase $\mathrm{YBa}_{2} \mathrm{Cu}_{3} \mathrm{O}_{6+\mathrm{x}}$ of this material [22-24].

Although the present work focuses on the $Y 123$ system, we note that other high- $T_{c}$ 's have been investigated via the 2D-ACAR spectroscopy [25-30]. Also, a large body of literature is devoted to Doppler broadening and lifetime studies of the high- $T_{c}$ 's, for recent work in this area see, e.g., Ref. 3133 , and citations therein.

An outline of this article is as follows. The introductory remarks are followed in Section II by an overview of the band structure theory based methodology for computing the electron-positron momentum density involved in the analysis of the 2D-ACAR spectra. Section III gives a brief overall discussion of the temperature dependent 2D-ACAR spectra in various phases of Y123. The metallic 
phase is considered in Section IV, including the c-axis projected momentum density (Section IV.1), the a-axis projection [34] (Section IV.2), and some remarks on the normal to superconducting transition. The insulating phase [35] and the metal/insulator transition are discussed in Section V, followed by a few concluding remarks.

\section{THEORETICAL OVERVIEW}

The spectral quantity of relevance in connection with the 2D-ACAR experiment is the electron-positron momentum density $\rho_{2 \gamma}(\mathbf{p})$, which can be expressed in the form

$$
\rho_{2 \gamma}(\mathbf{p})=\frac{1}{\pi^{2}} \int d \mathbf{r} \int d \mathbf{r}^{\prime} \exp \left[-i \mathbf{p} \cdot\left(\mathbf{r}-\mathbf{r}^{\prime}\right)\right] \int_{\times \operatorname{Im} G\left(\mathbf{r}, \mathbf{r}^{\prime} ; E\right) \operatorname{Im} G_{+}\left(\mathbf{r}, \mathbf{r}^{\prime} ; E_{+}\right)} d E f(E) \int d E_{+} f_{+}\left(E_{+}\right)
$$

in terms of the electron and positron one-particle Green's functions $G(E)$ and $G_{+}(E)$, and the associated Fermi-Dirac distribution functions $f(E)$ and $f_{+}(E)$ respectively. Note that Eq. (1) assumes independent particles and implicitly neglects the effects of electron-positron correlation (see e.g. [21]). The 2DACAR experiment measures the 2D-projection of $\rho_{2} \gamma(\mathrm{p})$ along a specific direction in momentum space.

Our theoretical treatment is based on the application of multiple-scattering theory techniques to a system of non-overlapping muffin-tin potentials (including 'empty' spheres) to represent the crystal potential. The form of $\rho_{2 \gamma}(p)$ for a general lattice with basis may be described most simply in terms of the formula for the electron momentum density $\rho(p)[36]$ :

$\rho(\mathbf{p})=-\frac{(4 \pi)^{2}}{\tau} \sum_{j} \frac{f(E)}{\left(E-p^{2}\right)^{2}} \frac{\mid \sum_{\mu} \exp \left(-\mathrm{ip} \cdot \mathbf{b}_{\mu}\left|\sum_{L} \bar{C}_{L \mu}(\mathbf{k}) s_{l \mu}(p, E) \cot \eta_{l \mu} Y_{L}(\mathbf{p})\right|^{2}\right.}{\sum_{\mu v} \sum_{L L^{\prime}} \bar{C}_{L \mu}(\mathbf{k}) \dot{M}_{L L^{\prime}}^{\mu \nu} \bar{C}_{L^{\prime} v}(\mathbf{k})}$

Here, all vectors and matrices are in the space of angular momentum and basis atom indices $L$ and $\mu$, and are to be evaluated at the energies $E=E_{\mathrm{k} j}$ of the Bloch levels at the reduced wave vector $\mathrm{k} . Y_{L}(\mathrm{p})$ is the $L$ th real spherical harmonic, $\tau$ the unit cell volume, $b_{\mu}$ denotes the position, and $\eta_{l} l$ the $l$ th phase-shift of the $\mu$ th atom. $s_{l} \mu(p, E)$ are smoothly varying functions involving the solutions of the radial Schrodinger equation for the $\mu$ th muffin-tin potential. The remaining quantites are the KKR matrix $M$ and its eigenvectors $C_{L \mu}(\mathrm{k})$. They satisfy the secular equation $M C=0$. The momentum density of the positron in its ground-state $(k=0)$ is given by a formula similar to Eq. (2), except that in this case there is no summation over $j . \rho_{2 \gamma}(\mathrm{p})$ is then obtained straightforwardly as a convolution of the $\mathrm{e}^{-}$and $\mathrm{e}^{+}$momentum density functions, see Ref. 36 for details.

\section{POSITRON SPECTRA IN Y123; AN OVERVIEW}

A typical as observed 2D-ACAR spectrum is shown in Fig. 1. Fig. 2 shows sections through the 2DACAR spectrum for both the metal $(x=6.9)$ and the insulator $(x=6.2)$ at low and high temperatures [35]. The electronic structure effects which are our primary concern here manifest themselves as structure in the 2D-ACAR spectra. Following common practice (see, e.g., [2]), such structure may be exposed by considering the anisotropic spectrum $A(p)$ defined by

$$
A(p)=M(p)-S(p)
$$

where $M(p)$ denotes the measured $2 D-A C A R$ and $S(p)$ is a smooth radially symmetric function. In the following, we discuss the nature of the electronic states, including the Fermi surface, in the metallic 
and insulating phases of $\mathrm{Y} 123$ in terms of the anisotropic spectra $A(p)$ which turn out to be relatively temperature independent.

Some comments concerning the large temperature effects seen in Fig. 2 are appropriate however. We see that as we go from the metallic to the insulating state, the 2D-ACAR becomes narrower or more peaked near $\mathrm{p}=0$ at both temperatures (all spectra are area normalized); the change at the metal/insulator transition is however about two times larger at $300 \mathrm{~K}$ than at $30 \mathrm{~K}$ near $\mathrm{p}=0$. The temperature effect, observed in going from $30 \mathrm{~K}$ to $300 \mathrm{~K}$, is similar for for the metal and the insulator, but the direction is opposite, i.e., in the metal the $30 \mathrm{~K}$ spectrum is narrower whereas in the insulator the $300 \mathrm{~K}$ spectrum is narrower.

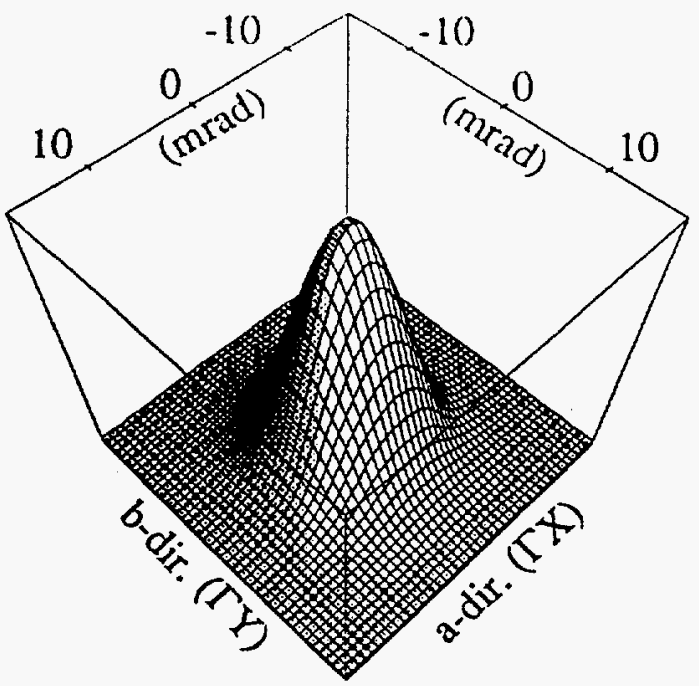

Fig.1 2D-ACAR data for $\mathrm{YBa}_{2} \mathrm{Cu}_{3} \mathrm{O}_{6.9}$ in c-axis projection (300K).

A variety of mechanisms are probably involved in the effects seen in Fig. 2. Since the positron annihilation experiment measures the electronpositron momentum density, the spectra reflect changes in the nature of both the positron and the electron states of the system (intrinsic effect), as well as possible changes in positron trapping (extrinsic effect) in the sample. While these questions are of interest in their own right, the key point to keep in mind for our present purpose is the aforementioned insensitivity of the anisotropic spectra $A(p)$ to temperature, suggesting that the effects of Fig. 2 are likely not intrinsic; see Ref. 11, 35 for a further discussion.

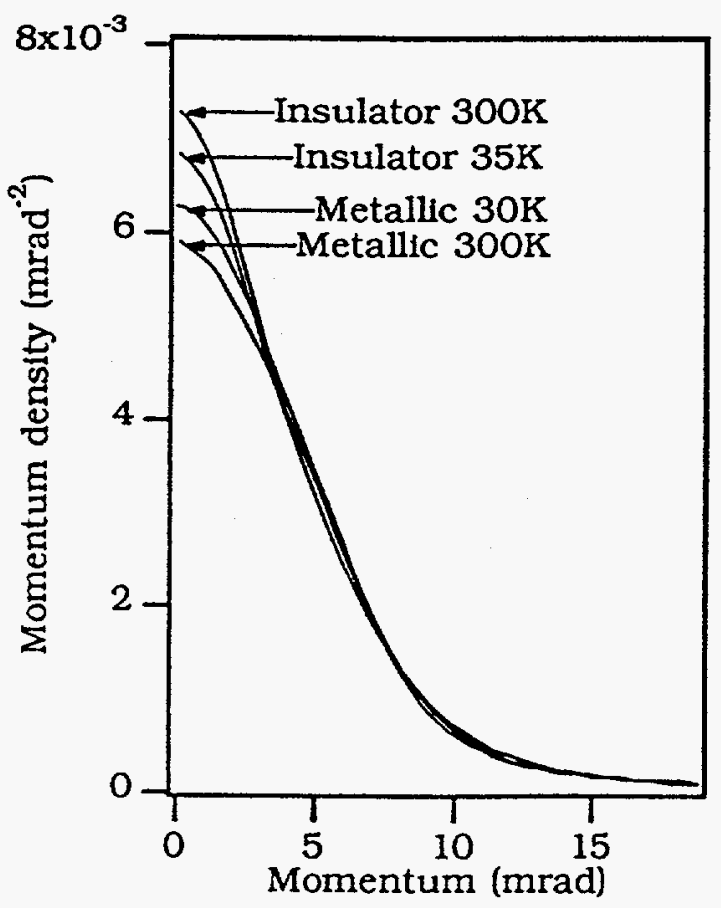

Fig. 2: Sections along the $\Gamma X$ direction through the c-projected 2DACAR spectrum of $\mathrm{YBa}_{2} \mathrm{Cu}_{3} \mathrm{O}_{6.2}$ at $35 \mathrm{~K}$ and $300 \mathrm{~K}$ (upper pair), and $\mathrm{YBa}_{2} \mathrm{Cu}_{3} \mathrm{O}_{6.9}$ at $30 \mathrm{~K}$ and $300 \mathrm{~K}$ (lower pair). All spectra are area normalized.
IV THE METALLIC PHASE

\section{IV.I THE C-PROJECTION}

The anisotropic distributions, shown in Fig. 3 at 30 and $300 \mathrm{~K}$, are rather similar in that these consist of a central ridge-like feature surrounded by four mountains. In addition, a less prominent sideridge along the $\Gamma \mathrm{X}$ direction is visible in both spectra at around 12 mrad; results in agreement with those of Fig. 3 (300K) have also been reported by Refs. 9 and 10.

The ridge feature and the associated umklapps (sideridges) are signatures of the one-dimensional Fermi surface, which is an electron sheet parallel to the a-c plane in the first zone, and originates from electron states in the chain region [37-39]. The four mountains, which exhibit $\mathrm{C}_{4 v}$ symmetry, are most likely in part caused by electron states in the $\mathrm{Cu}-\mathrm{O}$ planes, and in part by states in the chain area. These spectra do not show a clear imprint of the $\mathrm{Cu}-\mathrm{O}$ 

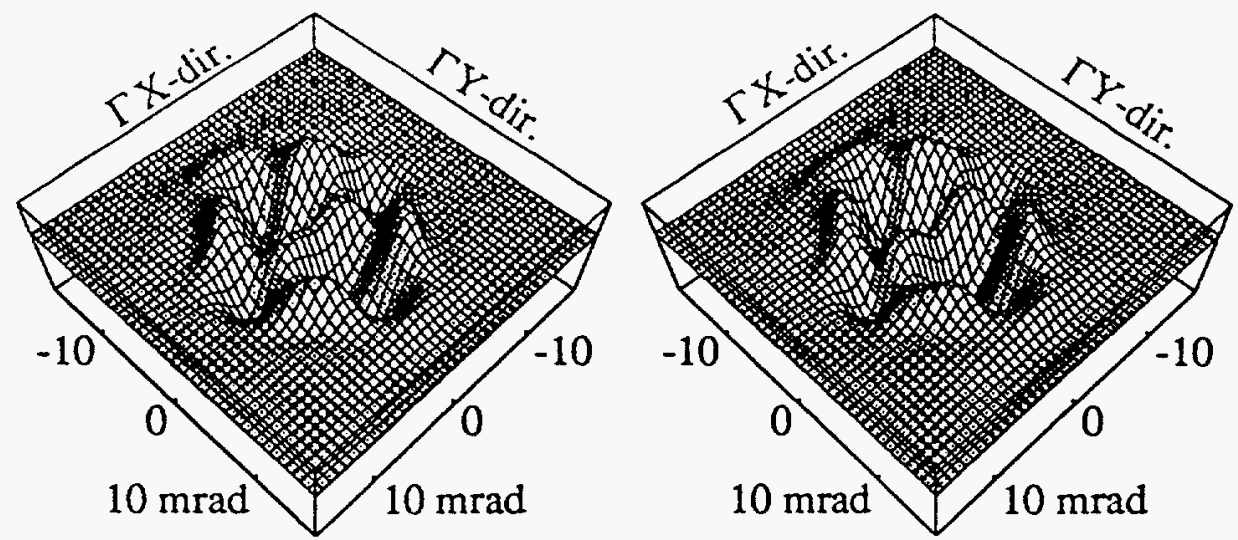

Fig. $3 \mathrm{~A}$ three-dimensional rendition of the anisotropic spectra $A(p)$ from untwinned $\mathrm{YBa}_{2} \mathrm{Cu}_{3} \mathrm{O}_{6.9}$ in c-axis projection at 30 (left) and $300 \mathrm{~K}$ (right).

plane Fermi surfaces; this is because the positron wave function overlap with the $\mathrm{Cu}-\mathrm{O}$ plane electrons is small compared to the overlap with electrons in the chain region [18-22,33].

In comparing band theory predictions with experimental results (for twinned samples), it was noted early that the shapes of the computed and measured 2D-ACAR's differ substantially, the theoretical results being flatter near $p=0$ in comparison to the measurements; this discrepancy is seen from Fig. 4 to be present for the untwinned specimen as well. In analyzing twinned data, Ref. 19 found that if an isotropic 'background" is subtracted from the data, then the theory and experiment agree in many details, leading to the suggestion that the experimental spectra should be corrected for background before making such a comparison. The physical origin of the background is not clear, but it arises presumably from defects, oxygen inhomogenieties and other effects not included in the computations that assume a perfect crystal. In Ref. 11 the temperature effect is reexamined and it is shown how a more satisfactory background correction can be made in terms of the temperature dependent spectra.

Fig. 5 compares the background corrected spectrum with the normalized theoretical spectrum along the high symmetry directions in the first as well as higher Brillouin zones. A reference to Figs. 4 and 5 shows a dramatic improvement regarding the overall agreement between the theory and experiment. To enhance FS signatures, anisotropies -- in the form of difference sections for several pairs of directions -are considered in Fig. 6 for the background corrected data. We comment on the theoretical results of Fig. 6(a) first. The complexity of these spectra arises not only from the presence of various $\mathrm{FS}$ sheets in $\mathrm{YBa}_{2} \mathrm{Cu}_{3} \mathrm{O}_{7}$, but also from the detailed character of the electronic wave functions of various filled bands. Several of the 'breaks' associated with the Fermi surface are indicated (e.g., $r_{1}, r_{2}, s_{1}, \ldots$, etc.). The sharpness of such breaks reflects the nearly dispersionless nature of the band structure of $\mathrm{YBa}_{2} \mathrm{Cu}_{3} \mathrm{O}_{7}$ along the c-axis.

The signatures of various FS sheets in Fig. 6(a) are as follows. The ridge FS gives the rapid variation $\left(\mathrm{r}_{0}\right)$ near $\mathrm{p}=0$; since the ridge lies along the $\Gamma X$ direction, in the $\Gamma S-\Gamma X$ spectrum or other difference spectra involving the $\Gamma$-point, the

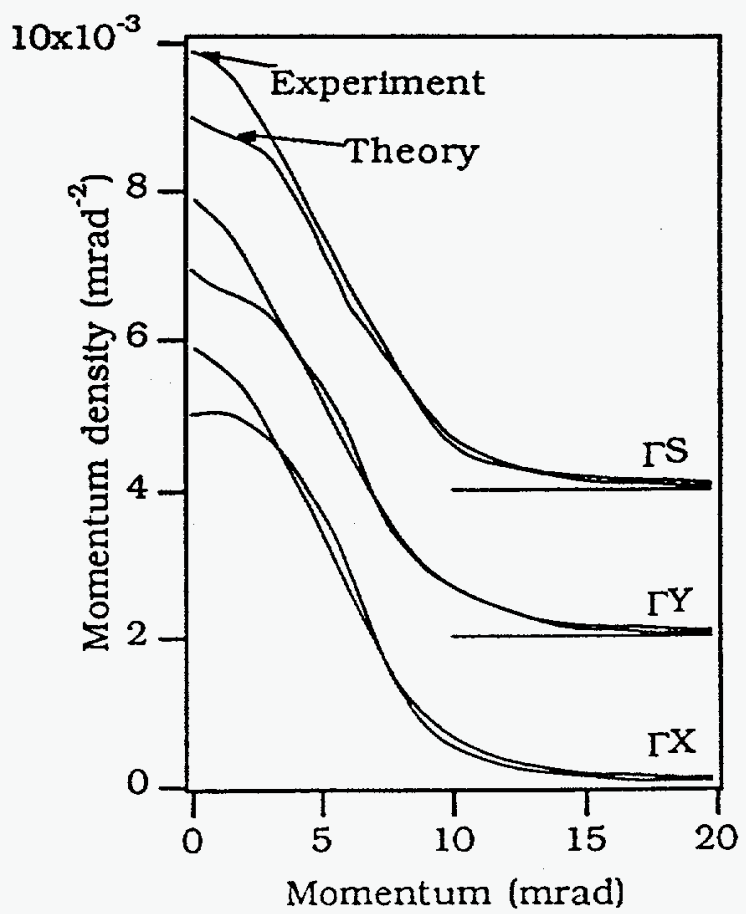

Fig.4 Comparison between theory and the as observed sections through typical 2D-ACAR spectra for untwinned $\mathrm{YBa}_{2} \mathrm{Cu}_{3} \mathrm{O}_{7-\mathrm{x}}$. The results are shown for the $300 \mathrm{~K}$ measurements. 


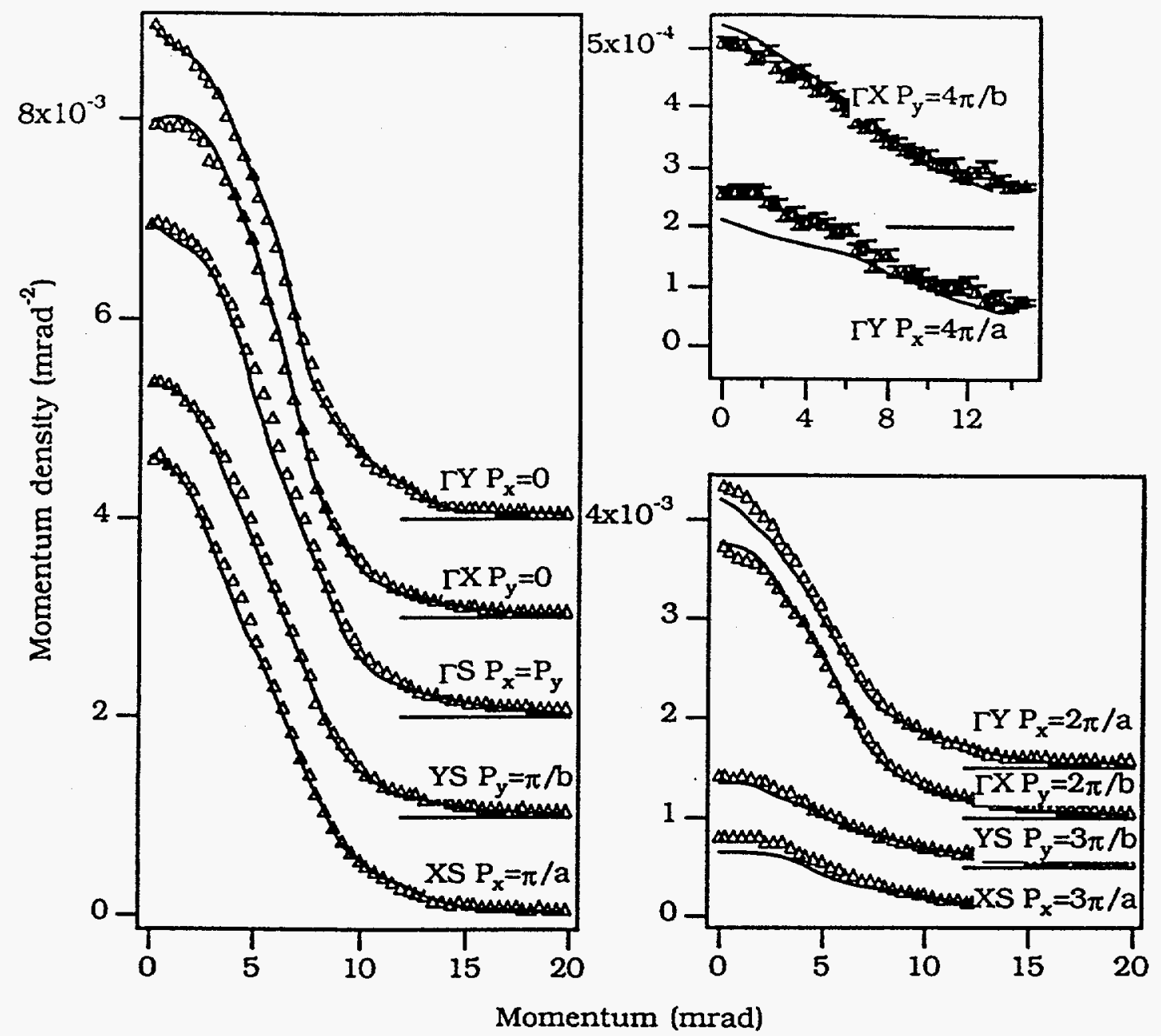

Fig. 5 A comparison of various sections through the background corrected 2D-ACAR spectrum for the untwinned $\mathrm{YBa}_{2} \mathrm{Cu}_{3} \mathrm{O}_{6.9}$ sample with the corresponding theoretical predictions of Ref.[19]. The results for the high symmetry lines $\Gamma Y, \Gamma X, \Gamma S, Y S$, and $\mathrm{XS}$ and parallel lines through higher zones are shown as marked.

anisotropy is nearly zero for momenta (around $p=0$ ) of the order of the width of the ridge. The first umklapp of the ridge $\left(r_{1}, r_{2}\right)$ is seen clearly around $6.3 \mathrm{mrad}$ as a peak in $\Gamma Y-\Gamma X$, and a dip in $\Gamma S-\Gamma Y$; only weak features are seen at this momentum in the $\Gamma S-\Gamma X$ or XS-YS curves. The second image of the ridge $\left(r_{3}, r_{4}\right)$ occurs around $13 \mathrm{mrad}$. The high momentum end $\mathrm{r}_{4}$ is generally more clearly visible than the low momentum feature $r_{3} ; r_{4}$ in particular coincides with the zero crossing in $\Gamma S-\Gamma Y$. The FS Scentered hole (the "pillbox") yields relatively prominent features only in the XS-YS spectrum, and the breaks $s_{1}, s_{2}, s_{3}, s_{4}, s_{5}$, and $s_{6}$ are all associated with the pillbox. Finally, as expected, the Cu-O plane sheets give essentially no discernible signatures in the 2D-ACAR's; this is due to the weak coupling of the positron Bloch state with the $\mathrm{Cu}-\mathrm{O}$ planes [18-22].

Fig. $6(\mathrm{~b})$ shows that experimental anisotropies are in good accord with theory regarding the detailed spectral features; also the agreement concerning the absolute magnitude of the overall undulations is good. In contrast, uncorrected data - although having much the same features as shown in Fig. 6(b) -have undulations that are about a factor of two too small. The level of discrepancies seen in Fig. 6(b) is common in even simpler materials in first principles comparisons between theory and experiment.

As noted above, the ridge FS gives three distinct features: (1) rapid variation in anisotropy near $p=0$, (2) an image around $6.3 \mathrm{mrad}$, and (3) a second image around $13 \mathrm{mrad}$. The experimental spectra display all three of these features. Although we do not expect any 'breaks' in the experimental data due to 

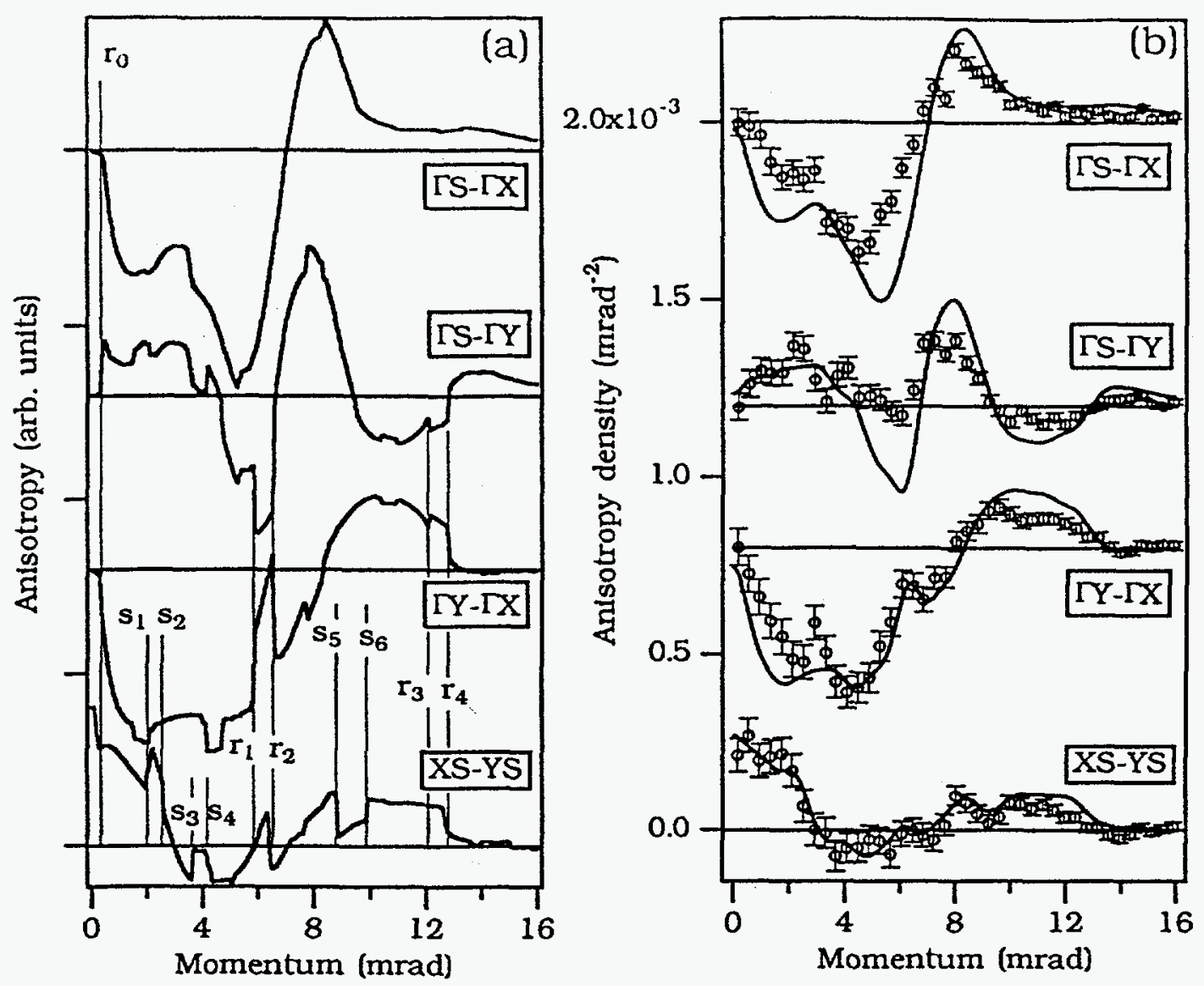

Fig. 6 (a): Theoretical anisotropic momentum distribution, here defined by taking differences between spectra along four different pairs of directions. Thin vertical lines mark the position of various FS features $r_{0}, s_{1}$, etc. discussed in the text. (b) A comparison between the resolution broadened theory curves in (a) with the corresponding experimental difference sections obtained from the corrected data. The spectra have been offset relative to one another as indicated by the horizontal lines.

resolution effects, near $p=0$, both sets of curves in Fig. $6(\mathrm{~b})$ possess similarly rapid variations and slopes; the width of the experimental ridge would however appear to be somewhat larger than that implicit in our band structure. The $6.3 \mathrm{mrad}$ image of the ridge $\left(r_{1}, r_{2}\right)$ is particularly important in the $\Gamma Y-\Gamma X$ curve because this feature lies on a relatively gently varying background and remains quite prominent in the theory even after including resolution broadening, and it is crucial that this feature is indeed observed clearly in the experimental data. By contrast, in the $\Gamma S-\Gamma Y$ curve, the resolution broadening effects would make the $6.3 \mathrm{mrad}$ ridge image difficult to distinguish from the background.

\section{IV.2 THE A-AXIS PROJECTION}

The above considerations all pertained to the $c$-axis projection. We now briefly consider results for the a-axis projection, which provides another view of the momentum density. Some current theoretical views hold that there may be fundamental differences in the description of the electron states (e.g., localized vs. itinerant) in the a- or b-directions compared to the c-direction [40]. In this regard, because an a-projection does not involve a momentum density integration in the c-direction, it is more sensitive to the electronic wave function character along the c-direction than is the c-projection. Also, since the cprojected momentum densities in $\mathrm{YBa}_{2} \mathrm{Cu}_{3} \mathrm{O}_{7}$ are in good agreement with band theory predictions, it would be interesting to establish whether the band theory continues to provide a reasonable description of the a-projected spectra. In Fig. 7 anisotropic data for a $\mathrm{c}$-axis and an a-axis projection both measured 

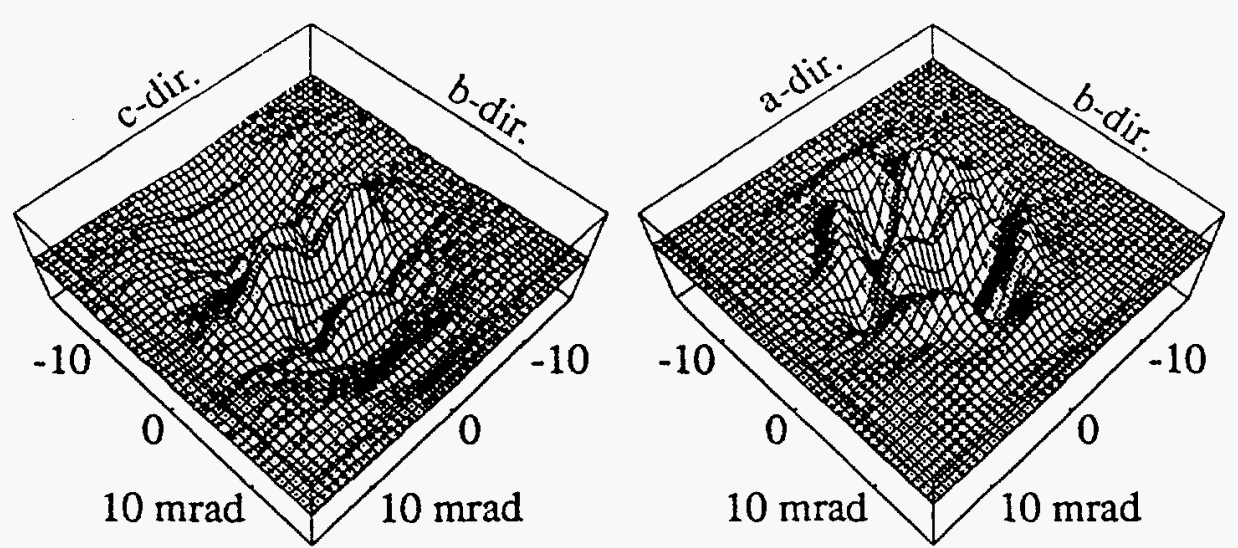

Fig. 7 A three-dimensional rendition of the anisotropic spectra $A(p)$ for untwinned $\mathrm{YBa}_{2} \mathrm{Cu}_{3} \mathrm{O}_{6.9}$. (a): in a-projection, and (b): in c-projection. The pixel size is 0.8 by 0.8 mrad.

at $300 \mathrm{~K}$ are compared [34]. We note that the present results for the a-axis projection appear similar to more recent results of Ref. 41 .

Fig. 7 allows us to delineate similarities and differences between the signature of the $\mathrm{Cu}-\mathrm{O}$ chain as revealed by the central ridge and its umklapp images in the two projections. The central ridge in both projections contains a minimum at $p=0$, and two peaks around $\pm 5 \mathrm{mrad}$, but in the a-projection, the anisotropic distribution extends to higher momenta and possesses additional peaks at $\pm 10 \mathrm{mrad}$. The 6 mrad first umklapp image of the ridge in the $\Gamma Y$ direction is clearer in the a-projection (ridge parallel to $\Gamma Z$ ); although hidden in the perspective view, this image is present also in the c-projection (ridge parallel to $\Gamma X)$, but the mountains overlap with this feature, reducing the momentum range over which it can be observed. Finally, the second umklapp image of the ridge around $13 \mathrm{mrad}$ is seen in both projections.

That the central ridge in both projections displays similar undulations in the $\Gamma \mathrm{Z}$ and $\Gamma X$ directions may at first sight appear surprising, because in the a-projection the ridge extends along the c-direction, while in the c-projection it extends along the a-direction, and the BZ dimensions in the c-and a-directions are widely different. This insensitivity of the momentum density to the tripling of the unit cell in the cdirection indicates that the electron wave functions approximately behave as if the lattice dimension in the c-direction were $1 / 3$ of the real lattice distance. The results of Fig. 7 are consistent with a picture where no dramatic change (e.g., itinerant vs. localized) takes place in the nature of the $\mathrm{Cu}-\mathrm{O}$ chain wave functions between the a- and c-directions. In particular, our results suggest that the electronic states are not strongly localized along the c-direction, although a detailed comparison with theoretical calculations is needed to reach a firm conclusion.

\section{IV.3 THE SUPERCONDUCTING TRANSITION}

In order to investigate the transition from the normal to the superconducting state around $91 \mathrm{~K}$, we have also measured c-projected 2D-ACAR spectra for the untwinned single crystal of $\mathrm{YBa}_{2} \mathrm{Cu}_{3} \mathrm{O}_{6.9}$ at $70 \mathrm{~K}$, $100 \mathrm{~K}$, and $185 \mathrm{~K}$. The spectra vary more or less uniformly with temperature and lie between the $30 \mathrm{~K}$ and $300 \mathrm{~K}$ results in a plot such as that of Fig. 2. A detailed comparison of the $70 \mathrm{~K}$ and $100 \mathrm{~K}$ data which bracket the superconducting transition does not reveal any substantial changes in either the 2DACAR or the anisotropic spectra $A(p)$, the latter appear similar to the data of Fig. 3. In this sense, the positron spectroscopy does not reveal significant changes in the electronic spectrum induced by pairing; we should keep in mind however that in Y123 the positron, as already noted, is more sensitive to the chain rather than the plane electrons. 

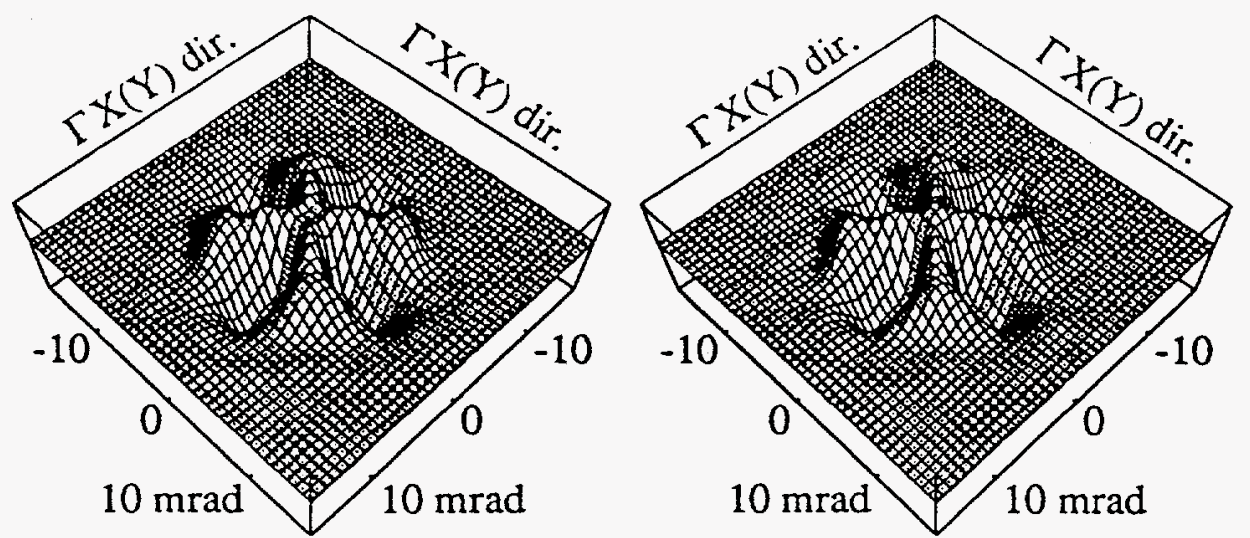

Fig. 8 The anisotropic 2D-ACAR spectra, A(p), for the insulator at $35 \mathrm{~K}$ (left) and $300 \mathrm{~K}$ (right).

\section{THE INSUlatiNG PHASE; THE METAL-INSULATOR TRANSITION}

Further insight into the electronic structure of $Y-123$ is obtained by augmenting the studies of the metallic phase with those of the insulating phase $\mathrm{YBa}_{2} \mathrm{Cu}_{3} \mathrm{O}_{6+x}[22-24,35]$. The close combination of band theory and experiment that has lead to the understanding the 2D-ACAR spectra in the metallic $Y$ 123 is less likely to succeed for the insulator, since the traditional band theory encounters well-known difficulties in describing the ground state of the insulating oxides. In the following we compare results from a c-axis projection of $\mathrm{YBa}_{2} \mathrm{Cu}_{3} \mathrm{O}_{6.2}$ insulating sample measured at $35 \mathrm{~K}$ and $300 \mathrm{~K}$ to those of the metallic phase [35]. We note that our results appear similar to those reported at $40 \mathrm{~K}$ in Ref. 22.

The anisotropic spectra of Fig. 8 and Fig. 3 allow us to delineate similarities and differences between the character of momentum density in the insulating and metallic phases of Y-123. A substantial overall change in the structure of the anisotropic 2D-ACAR spectra is seen to be induced by the metal/insulator transition at both the low and the high temperature. The most striking difference between the insulator and the metal is that of symmetry, the metal being orthorhombic and therefore resulting in a projection with $\mathrm{C}_{2 \mathrm{v}}$ symmetry, while the tetragonal insulator is $\mathrm{C}_{4 \mathrm{v}}$ symmetric. In the insulator the four mountain like features are present, but the central ridge and the side-ridges (see Fig. 3) are missing (see also [24]); a new feature connecting the four mountains in the shape of a 'cross' centered on $\mathrm{p}=0$ makes its appearance.

Some of the differences seen between Fig. 8 and Fig. 3 may be understood by recalling that the positron preferentially samples the chains in the 07 phase, and that the removal of $O$-atoms from the chains tends to localize the positron state in this area of the unit cell. We therefore expect that as the metallic, nearly $\mathrm{O}$, sample is deoxygenated and the $\mathrm{Cu}-\mathrm{O}$ chains are disrupted, the signatures of the $\mathrm{Cu}-\mathrm{O}$ chains in the 2D-ACAR will rapidly disappear due not only to the removal of the chains themselves but also due to a reduced overlap with the positron wave-function. These conclusions are consistent with the insulator spectra of Fig. 8, where we see the absence of the central ridge like structures extending along $\Gamma X$ (or $\Gamma Y$ ) directions, along with the absence of the side-ridges. The remaining major feature of the $\mathrm{O} 7$ $2 D-A C A R$, namely the four mountains along $\Gamma S$, arise from various filled states including those associated with $\mathrm{Cu}-\mathrm{O}$ planes and chains, and appear to be insensitive to the removal of chains. Finally, the new feature in the insulator, the aforementioned 'cross', appears to have no simple explanation in terms of the metallic 2D-ACAR.

The anisotropic spectra of Fig. 8 obviously contain what appear to be quite sharp structures. It turns out however that much of this structure lies close to Brillouin zone boundaries, and cannot therefore be taken to represent Fermi surfaces (sce also [42]). Based on this and other arguments, Ref. [35] concludes that no Fermi surface signatures can be inferred in the 2D-ACAR spectra of insulating $Y 123$. 
Finally, we compare in Fig. 9 the anisotropy in the form of difference between the $\Gamma S$ and $\Gamma X$ sections for the insulator with the corresponding theoretical results of Ref. 20. While the use of a paramagnetic metallic band structure, implicit in the work of Ref. 20, to obtain electron-positron momentum density in O6, which is an antiferromagnetic insulator, is not satisfactory, the computation is nevertheless seen to capture some of the main features of the experimental data. The $\Gamma S-\Gamma X$ anisotropy is seen to change dramatically at the metal/insulator transition; the metal contains (Fig. $6 ; \Gamma S-\Gamma X$ section) a broad negative lobe followed by a positive peak around $8 \mathrm{mrad}$, whereas the insulator gives two positive peaks centered around 4 and $8 \mathrm{mrad}$. Despite some discrepancies, the overall agreement between the theory and experiment in the insulator is surprisingly good, and as noted by Ref. 20, may reflect the lack of a significant overlap between the positron wave function and the $\mathrm{Cu}-\mathrm{O}$ plane related bands which in the band theory incorrectly turn out to be metallic.

\section{CONCLUSIONS}

We have presented positron annihilation (2D-ACAR) results for the three fundamental phases of $\mathrm{YBa}_{2} \mathrm{Cu}_{3} \mathrm{O}_{7-x}$, namely, the normal metal, superconductor, and the insulator. A clear signature of the Fermi surface associated with the one-dimensional $\mathrm{Cu}-\mathrm{O}$ chain band is found in both the normal and the superconducting metallic state. The a-axis projected spectrum is consistent with a picture where no dramatic change (e.g., itinerant vs localized) takes place in the nature of at least the $\mathrm{Cu}-\mathrm{O}$ chain wave functions between the a- and c-directions. No Fermi surfaces could be identified in the spectra from the insulator. Detailed comparisons are made between the measured 2D-ACAR's with the corresponding first-principles band theory predictions, and an impressive level of agreement is found in the metallic phase; surprisingly, the band theory appears to capture some major features of the observed spectra from the insulator, eventhough the band theory incorrectly predicts $\mathrm{YBa}_{2} \mathrm{Cu}_{3} \mathrm{O}_{6}$ to be metallic.

Finally, we note that the 2D-ACAR spectra show substantial temperature effects in the metallic as well as the insulating samples, large changes are evident at the metal/insulator transition but not at the superconducting transition. However the important structure in the spectra, as revealed via the anisotropies in the 2D-ACAR which are the most relevant aspects of the spectra for delineating the electronic structure and Fermiology, are relatively insensitive to temperature. Our analysis suggests that the temperature effects are most likely extrinsic in nature (shallow positron traps, oxygen inhomogeneities, etc.), and do not reflect the character of the electronic states of the 'ideal' Y123.

\section{ACKNOWLEDGMENTS}

The authors benefitted from discussions with R. Benedek and P.E. Mijnarends. The present work was supported by the U.S. Department of Energy, Basic Energy Sciences, Division of Materials Sciences under contract \#W-31-109- ENG-38, including a subcontract to Northeastern University $(A B)$, and a travel grant from NATO. This work benefited from the allocation of supercomputer time on the NERSC and San Diego Supercomputer centers.

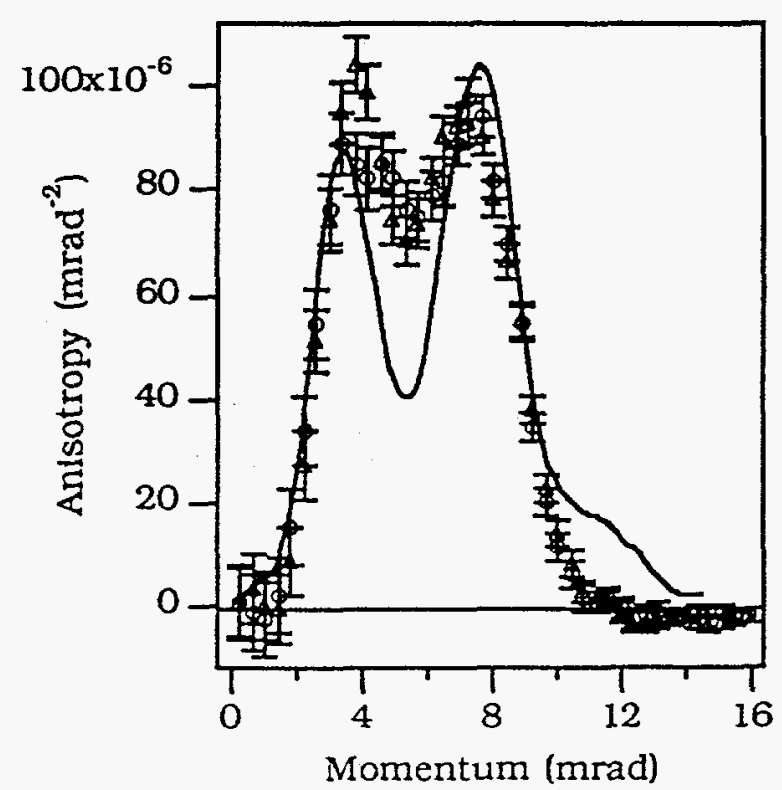

Fig. 9 The experimental difference section $\Gamma S-\Gamma X$ in the insulator at $35 \mathrm{~K}$ (triangles) and at $300 \mathrm{~K}$ (circles). The corresponding theoretical results of Ref. 20 for $\mathrm{YBa}_{2} \mathrm{Cu}_{3} \mathrm{O}_{6}$ (solid line) are scaled arbitrarily to obtain visual agreement with the experimentil data. 
REFERENCES

[1] M. Peter. L. Hoffmann, and A.A Manuel Physica C 153-1551724 (1988).. L. Hoffmann. A.A. Manuel, M. Peter, E. Walker, and M.A. Damento Europhys Lett. 661 (1988)., and Physica C 153 155129 (1988). M. Peter and A.A. Manuel, Phys. Scripta T29, 106 (1989)

[2] L.C. Smedskjaer. J.Z. Liu, R. Benedek, D.G. Legnini. D.J. Lam, M.D. Stahulak, H. Claus , and A. Bansil Physica C 156269 (1988).

[3] H. Haghighi, J.H. Kaiser, S. Rayner, R.N. West, M.J. Fluss, R.H. Howell, P. E. A. Turchi, A.L. Wachs, Y.C. Jean, and Z.Z. Wang J. Phys. Condens. Matter 21911 (1990).

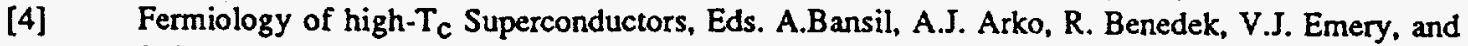
L.C. Smedskjaer, J. Phys. Chem. Solids 52 , issues 11/12 Dec. 1991.

[5] E. Abrahams J. Phys. Chem. Solids 52. 1595, (1991)

[6] M. Peter J. Phys. Chem. Solids $\underline{52}$, 1599, (1991)

[7] L. C. Smedskjaer, A. Bansil, U. Welp. Y. Fang, and K. G. Bailey, J. Phys. Chem. Solids 52, 1541,(1991)

[8] L. Hoffmann, W. Sadowski, A. Shukla, Gh. Adam, B. Barbiellini, and M. Peter, J. Phys. Chem. Solids 52, 1551,(1991).

[9] L. Hoffmann . W. Sadowski, and M. Peter, Proceedings of the 9 'th "Int. Conf. on Positron Anrihilation", Hungary (1991)

[10] H. Haghighi, J.H. Kaiser, S. Rayner, R.N. West, J.Z. Liu, R. Shelton, R.H. Howell, F. Solar, and M.J. Fluss, J. Phys. Chem. Solids 52, 1535,(1991) and Phys. Rev. Lett., 67, 382, (1991)

[11] L.C. Smedskjaer, A. Bansil, U. Welp, Y. Fang, and K.G. Bailey, Physica C 192, 259 (1992); L.C. Smedskjaer, A. Bansil. Proceedings of the 9'th "Int. Conf. on Positron Annihilation", Hungary (1991).

[12] J.C. Campuzano, G. Jennings, A.J. Arko, R.S. List, B.W. Veal, and R. Benedek, J. Phys. Chem. Solids 52. 1411,(1991); J.C. Campuzano, L.C. Smedskjaer, R. Benedek, G. Jennings, and A.Bansil, Phys. Rev. B 43, 2788 (1991); J.C. Campuzano, G. Jennings, M. Faiz, L. Beaulaigue, B.W. Veal, J.Z. Liu, A.P. Paulikas, K. Vanderwoort, H. Claus, R.S. List, A.J. Arko, and R.J. Bartlett, Phys. Rev. Lett. 64, 2308 (1990).

[13] C.G. Olson, J.G. Tobin, F.R. Solal, C. Gu, J.Z. Liu, M.J. Fluss, R.H. Howell, J.C. OBrien, H.B. Radousky, and P.A. Sterne, J. Phys. Chem. Solids 52, 1419.(1991); J.G. Tobin, C.G. Olson, C. Gu, J.Z. Liu, F.R. Solal, M.J. Fluss, R.H. Howell, J.C. OBrien, H.B. Radousky, and P.A. Sterne, Phys. Rev. B 45.5563 (1992).

[14] T. Takahashi, T. Watanabe, T. Kusunoki, and H. Katayama-Yoshida J. Phys. Chem. Solids 52, 1427, (1991)

[15] R. Liu, B.W. Veal, A.P. Paulikas, J.W. Downey, H. Shi, C.G. Olson, C. Gu, A.J. Arko, J.J. Joyce, and R.J. Bartlett. J. Phys. Chem. Solids 52, 1437.(1991); Ibid. Phys. Rev. B 45,5614 (1992)

[16] C. M. Fowler, B. L. Freeman, W. L. Hults, J. C. King. F. M. Mueller, and J.L. Smith. Phys Rev. Lett. 68, 534 (1992); F. M. Mueller, J. Phys. Chem. Solids 52, 1457 (1991); J.L. Smith, C. M. Fowler, B. L. Freeman, W. L. Hults, J. C. King, and F. M. Mueller to appear in Proc.. of third Int. Symp.. on Superconductivity, Sendai Japan.

[17] G. Kido, K. Komorita, H. Katayama-Yoshida, and T. Takahashi, J. Phys. Chem. Solids 52, 1465, (1991)

[18] A. Bansil, R. Pankaluoto, R.S. Rao, P.E. Mijnarends, R. Prasad, and L. C. Smedskjaer Phys. Rev. Lett. 612480 (1988); A. Bansil „J. Phys. Chem. Solids 52. 1493,(1991); A. Bansil, P.E. Mijnarends, and L.C. Smedskjaer Phys. Rev. B 43,3667 (1991).

[19] A. Bansil, P.E. Mijnarends, and L.C. Smedskjaer, Physica C 172175 (1990).

[20] S. Massida, Physica C 169.137 (1990); S.Massida, Jaejun Yu, A.J. Freeman, L. Hoffmann, P. Genoud, and A.A.Manuel, J. Phys. Chem. Solids 52, 1503,(1991)

[21] T. Jarlborg. B. Barbiellini, E. Boronski, P. Genoud, and M. Peter, J. Phys. Chem. Solids $\underline{52} 1515$, (1991)

[22] B. Barbiellini, P.Genoud, J.Y. Henry, L. Hoffmann, T. Jarlborg, A.A. Manuel, S. Massida, M. Peter, W. Sadowski, H.J. Scheel. A. Shukla, A.K. Singh, and E. Walker Phys Rev B 43,7810 (1991).

[23] M. Peter, L. Hoffmann, and A.A. Manuel in Pesitron Annihilation, World Scientific (1989), eds. L. Dorikens-Vanpraet, M. Dorikens, and D. Segers, Proceedings of ICPA-8, pp. 197

[24] M. Peter, A.A. Manuel, L. Hoffmann and W. Sadowski, Europhys. Lett. 18, 313, (1992)

[25] P.E. Mijnarends, A.F.J. Melis, and A.W. Weeber, J. Phys. Chem. Solids 52, 1569, (1991); P.E. Mijnarends, A.F.J. Melis, A.W. Weeber, A.A. Menowsky, and K. Kadowaki Physica C 176,113 (1990) 
[26] L.P. Chan, D.R. Harshman, K.G. Lynn, S. Massida, and D.B. Mitzi, J. Phys. Chem. Solids 52 ,

[27] L. P. Chan. K.G. Lynn. D.R. Harshman, L.R. Monar, S. Massida, Jacjun Yu, A.J. Freemann, C.P. Tigges, B. Morosin, D.S. Ginley, and E.L. Venturini, Bull. Am. Phys. Soc. 37 . 336 (1992).

[28] R. Howell, P.A. Sterne, F. Solal, M.J. Fluss, J.H. Kaiser, R.N. West, K. Kitazawa, and H. Kojima Bull. Am. Phys. Soc. 37. 336 (1992); R. Howell et al Proceedings of the 9'th "Int. Conf. on Positron Annihilation", Hungary (1991); P.E.A. Turchi, A.L. Wachs, K.H. Wetzler, J.H. Kaiser, R.N. West, Y.C. Yean, R.H. Howell, and M.J. Fluss, J. Phys. Cond. Matter. 2, 1635 (1990)

[29] P. Blandin, S. Massida, B. Barbiellini, T. Jarlborg, P. Lerch, A.A. Manuel, L. Hoffmann, M. Gauthier, W. Sadowski, E. Walker, M.Peter, Jaejun Yu, and A.J. Freeman, Phys. Rev. B 46 (to appear) (1992).

[30] S. Tanigawa, Y. Mizuhara, Y. Hidaka, M. Oda, M. Suzuki, and T. Murakami, Mater. Res. Soc. 5,57 (1988)

[31] A.A. Manuel J. Phys. Cond. Matter 1 SA107 (1989)

[32] Proceedings of the 9'th "Int. Conf. on Positron Annihilation", Hungary (1991)

[33] A. Bharati, C.S. Sundar, and Y. Hariharan, J. Phys. Cond. Materials 1. 1467 (1989)

[34] L.C. Smedskjaer, A. Bansil, U. Welp. Y. Fang, and K.G. Bailey, Bull. Am. Phys. Soc. 37336 (1992); Ibid. Phys Rev. B (1992)

[35] L.C. Smedskjaer, A. Bansil, A.P.Paulikas, B.W. Veal, D.G. Legnini, and K.G. Bailey, Physica C (1992)

[36] P.E. Mijnarends and A. Bansil, J. Phys.: Conden. Matter 2, 911 (1990)

[37] J.Yu, S.Massidda, A.J.Freeman, and D.D.Koelling, Phys. Lett. A122, 203 (1987)

[38] W. E. Pickett, R. E. Cohen, and H. Krakauer, Phys. Rev. B42, 8764 (1990)

[39] C.O. Rodriguez, A.I. Liechtenstein, I.I. Mazin, O. Jepsen, O.K. Andersen, and M. Methfessel, Phys. Rev. B 422692 (1990)

[40] Discussions at the Argonne Fermiology Workshop , March (1991); P.W. Anderson (to be published).

[41] L. Hoffmann et al., private comm. (1992)

[42] J. Friedel and M. Peter Europhys. Lett. ㅇ. 79 (1989) 